\title{
Analog Controlled Adaptive Feedforward Amplifier for IMT-2000 Band
}

\author{
Young-Jean Song, In-Ju Oh, Kyu-Jae Seo, Yong-Chae Jeong, Chul-Dong Kim* \\ Dept. of Information \& Communication Engineering Chonbuk National University, 664- \\ 14 Duchjin-Dong Duckjin-Gu Chonju Korea 561-756, ycjeong@moak.chonbuk.ac.kr \\ *Sewon Teletech Inc., 1023 Kwanyang-Dong Dongan-Gu Anyang Kyounggi Korea \\ Email: ycjeong@moak.chonbuk.ac.kr
}

\begin{abstract}
A novel adaptive design for feedforward amplifier linearizer with analog controller is proposed in this paper. Compared previous adaptive linearizing method, this is new design which does not require any pilot signal and intentional signal distortion. In main signal cancellation loop, magnitude and phase of two-path signal in subtracter are compared mutually and controlled for main signal cancellation. In distortion signal cancellation loop, gain and phase of each path is controlled to maintain constant, so that the same level and out-of phase of two-path distortion signal are obtained. A prototype feedforward amplifier with the proposed linearizer has been tested for 3-carrier IS-95 CDMA signals and 20.25dB ACPR is improved.
\end{abstract}

\section{INTRODUCTION}

In the third generation $(3 \mathrm{G})$ of mobile radio standards, very high linear transmitter that can support high crest factor signal is demanded than ever. So to power amplifier designer, high linearity and high efficiency is critical issue. In fact, as the power amplifier operates close to saturation region where both high efficiency and high output power emission are achieved, the degradation of linearity becomes significant. Compromise between power efficiency and linearity must be considered. Or linearization technique to recuperate nonlinearity of power amplifier is only solution. Various linearization methods which are named feedforward, feedback, predistortion, LINC(LInear amplification with Nonlinear Components), CALLUM(Combined Analog Locked Loop Universal Modulator), EER(Envelope Elimination and Restoration), and so forth are reported (1).

Among numerous amplifier linearization techniques, feedforward linearization has been extensively used in base-station amplifier because of its intrinsic advantages on providing high linearity over a wide frequency band and more tight specifications of nonlinearity in base station transmitter than mobile station transmitter. Since the linearizing parameters change dramatically due to varying operating conditions such as temperature, input power level and supply voltage, an adaptive control circuitry is essential. At now, several adaptive control approaches have been proposed. Fixed pilot tone method (2), pilot tone hopping method (3), the gradient method (4)(5), intentional signal perturbation method (6) are reported.

In this paper, analog controlled adaptive feedforward amplifier linearizing method to reduce distortion signal is derived. This method adjusts the circuit parameter adaptively for varying operating conditions. Feedforward linearizer consists of main signal cancellation loop and distortion signal cancellation loop. In main signal cancellation loop, magnitude of two input path signals in subtracter and relative phase of two input path signals to input carrier are compared mutually and controlled so that main signal can be cancelled at output port of subtracter. In distortion signal cancellation loop, distortion signals of subtracter output port are controlled continuously to have constant gain and phase so that distortion signal is cancelled at final port.

\section{ARCHITECTURE AND OPERATING PRINCLES}

The fundamental structure of feedforward amplifier linearizer consists of two signal cancellation loops. Based on the assumption that amplifier output signals are sum of amplified input signal and distortion signal. The amplified input signal is cancelled in main carrier cancellation loop, which leaves only distorted signal. In distortion signal cancellation loop, the distorted signal is amplified and cancelled at the amplifier output, which only leaves amplified input signal as the final output 
signal. Though theoretically feedforward structure can provide completely distortion free output signal, the actually achievable output is dependent on imbalance of amplitude, phase and delay time of each path signal that consist of loop.

Figure 1 shows schematic of the proposed analog controlled feedforward amplifier. Input signal go through divider, variable attenuator $\left(\mathrm{A}_{1}\right)$, variable phase shifter $\left(\Phi_{1}\right)$ and main amplifier. Some of amplified input signal at output port of main amplifier is extracted and connected to signal subtracter. Also input signal go through time delay line and is connected to subtracter. In subtracter, some of two-path signals are divided by dividers and go through RF switch (RF SW1). Others go through signal canceller that is realized hybrid coupler. RF switch can connect one of two paths by control clock signal. Output signal of RF switch is divided with divider (D1) and one of them is converted signal voltage by detector (DET1). Signal voltage is connected one port of magnitude comparator (MC). If RF switch (RF SW1) connect the other path, other path signal can be converted other signal voltage. So amplitude data of two-path signal in subtracter can be obtained. Magnitude comparator makes control voltage $\left(\mathrm{V}_{\mathrm{A} 1}\right)$ of variable attenuator $\left(\mathrm{A}_{1}\right)$ so that two-path signal voltage is matched. Magnitude comparators consist of inverting amplifier and integrator that can be realized with operational amplifiers.

The other output signal of divider (D1) is connected to RF port of IQ demodulator whereas a part of input signal is connected to LO port of IQ demodulator. Outputs of IQ demodulator are in-phase and quadrature-phase direct voltages that represent phase data between $\mathrm{LO}$ and $\mathrm{RF}$ port signal because of the same frequency spectrum. According to connection path of RF switch (RF SW1), phase data of two path signals in subtracter can be obtained. Phase comparator (PC) consists of twomagnitude comparators and controls voltage $\left(\mathrm{V}_{\Phi 1}\right)$ of variable phase shifter $\left(\Phi_{1}\right)$ so that phase of two-path signal in subtracter makes out-of-phase. Figure 2 shows schematic of manufactured IQ demodulator. The phase of general variable attenuator is changed as the attenuation level is changed. But, in this paper the phase variation of the used attenuator is minimized on attenuation. PIN diode for attenuator is used and external open stub transmission line compensates internal parasitic components of PIN diode that cause phase change. For good reflection coefficient, reflective type is adopted (7).

Whereas direct frequency spectrum comparison is possible in main signal cancellation loop because twopath signal have similar frequency spectrum, but frequency spectrum comparison is very difficult in the distortion cancellation loop because frequency spectrum of two-path is dissimilar. Signals in output port of main time delay circuit are sum of amplified input signal and distortion signal, but signal that pass through subtracter is just distortion signal. So direct comparison of two-path signal in distortion signal cancellation loop is impossible to cancel distortion signal. But if two-path signal can maintains constant gain and phase in spite of changing operating condition, the same level and out-of phase of two-path distortion signal are obtained, so that distortion signal can be cancelled. Usually insertion loss characteristic of delay line is almost constant, but phase characteristic is changed due to operating temperature. By using variable phase shifter $\left(\Phi_{3}\right)$ that is controlled by single supply centigrade temperature sensor, phase of distortion signal tracks out-of-phase with distortion signal of main path.

Signal in output port of subtracter go through divider $\left(D_{2}\right)$, variable attenuator $\left(A_{2}\right)$, variable phase shifter $\left(\Phi_{2}\right)$ and error amplifier. Most of amplified distortion signal is connected to combiner and a part of it is connected divider (D4) and go through automatic level controller (ALC) and RF switch (RF SW2). A part of subtracter output signal also connected other automatic level controller and RF switch (RF SW2). Output signals of $\mathrm{RF}$ switch are converted signal voltage and signal voltages are compared in magnitude comparator. Magnitude Controller (MC) controls attenuation voltage $\left(\mathrm{V}_{\mathrm{A} 2}\right)$ of attenuator $\left(\mathrm{A}_{2}\right)$ so that constant gain is achieved (8).

Since automatic level controller consists of low phase shifting attenuator and detector, there is little phase variation on automatic level control process. Output signals of subtracter and error amplifier are combined with combiner. The level of combined signals is different by phase difference between two signals. Hence detection voltage of combined signals is also different. That is, detection voltage shows phase difference between signals. If magnitude comparator compares detection voltage with fixed voltage and controls voltage $\left(\mathrm{V}_{\Phi_{2}}\right)$ of variable phase shifter $\left(\Phi_{2}\right)$ to maintain detection voltage, phase of this path would be maintained constant. Figure 3 shows idea of phase controller.

\section{EXPERIMENTS AND RESULTS}

To show validation of the proposed linearizing method, proto-type analog controlled adaptive feedforward amplifier for IMT-2000 base-station transmitting band was fabricated. The line-up of main amplifier was ERA5SM of Mini-Circuits and MHL-21336 of Motorola. The gain and $\mathrm{P}_{1 \mathrm{~dB}}$ of main amplifier was $47 \pm 0.1 \mathrm{~dB}$ and $34.7 \mathrm{dBm}$, respectively. The line-up of error amplifier was two ERA-5SM and an MHL-21336 of Motorola and the gain was $53 \pm 0.1 \mathrm{~dB}$. Low phase shifting variable attenuator was realized with reflective type and $\pm 0.15^{\circ}$ 
phase shifted on $20 \mathrm{~dB}$ attenuation. Used PIN diode was HSMP-4810 of HP. Variable phase shifter was realized with reflective type for good reflection characteristic and $55^{\circ}$ phase was shifted on $12 \mathrm{~V}$ variation. Used varactor diode was 1T362 of Sony.

The 3-carrier IS-95 CDMA base station transmitting signal is used as input signal that center frequency is $2140 \mathrm{MHz}$. Main signal cancellation is achieved 21.1 $28.4 \mathrm{dBc}$ for average output power range $18.3 \sim 28.7 \mathrm{dBm}$. Main carrier cancellation results according to output power variation are shown at figure 4 . Figure 5 shows main signal cancellation result when output power level is $25.5 \mathrm{dBm}$. With proposed method, ACPR characteristic of amplifier is improved about $6.0 \sim 20.25 \mathrm{~dB}$. Figure 6 shows linearization result when output power level is $24.9 \mathrm{dBm}$. And figure 7 shows adaptive linearization results for output power range $15.7 \sim 26 \mathrm{dBm}$.

\section{CONCLUSIONS}

We have presented new analog controlled adaptive feedforward amplifier linearizer. In main signal cancellation loop, magnitude and phase of two-path signal in subtracter are compared and controlled mutually for main signal cancellation. In distortion signal cancellation loop, gain and phase of each path is controlled to maintain constant, so that the same level and out-of phase of two-path distortion signal are obtained. Hence adaptive linearization is achieved on varying operating conditions. With the proposed method, ACPR is improved $20.25 \mathrm{~dB}$ for 3-carrier IS-95 CDMA base station transmitting signal.

\section{ACKNOWLEDGEMENT}

This work was supported by grant No. 2000-1-30200007-3 from the Basic Research Program of the Korea Science \& Engineering Foundation.

\section{REFERENCES}

(1)Peter B. Kennington, High Linearity RF Amplifier Design, Artech House, 2000.

(2)D. L. Tatterstall, "Feed Forward Amplifer Network with Frequency Swept Pilot Tone," U.S Patent. No. 5,130,663, 1992.

(3)M. G. Choi, Y. C. Jeong, I. H. Park, "Method and Apparatus for Amplifying Feedforward Linear Power Using Pilot Tone Hopping," U.S. Patent. No. 6,081,156, 2000.

(4)R. H. Bauman, "Adaptive feed-forward system," U.S. Patent, 4,389,618, 1983.
(5)J. K. Cavers, "Adaptation behavior of a feedforward amplifier linearizer," IEEE Trans. Vehicular Tech., vol. 44, no. 1 , pp.31-40, 1995

(6)M. G. Overmann, J. F. Long, "Feedforward Distortion minimization circuit," U.S. Patent, 5,077,532, 1991

(7)Y. C. Jeong, "Design of A Novel Vector Modulator," Microwave Journal, vol.44, no.10, pp.156 162, Oct. 2001

(8)I. H. Kang, I. K. Chang, Y. C. Jeong, S. W. Yun, "The Design of Automatic Gain and Phase Controlled Amplifier," APMC Digest, pp.18-21, 1995

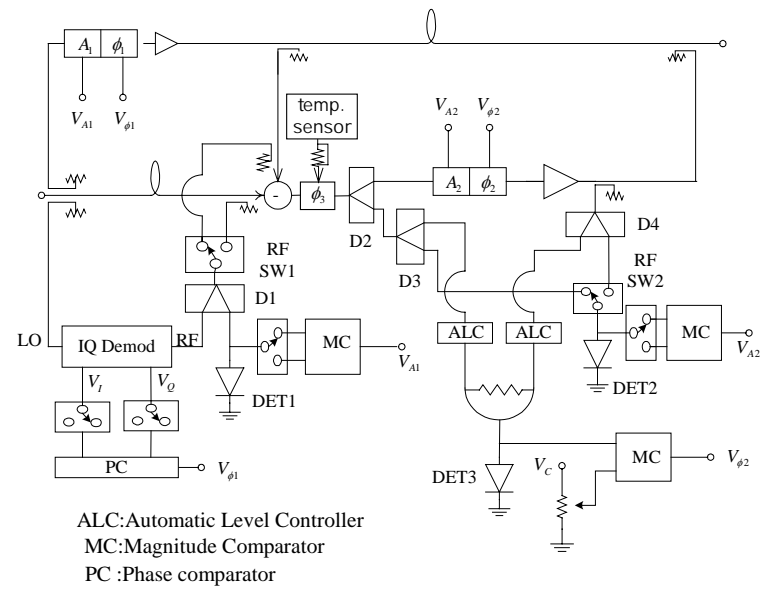

Figure 1: Schematic of proposed analog controlled adaptive amplifier

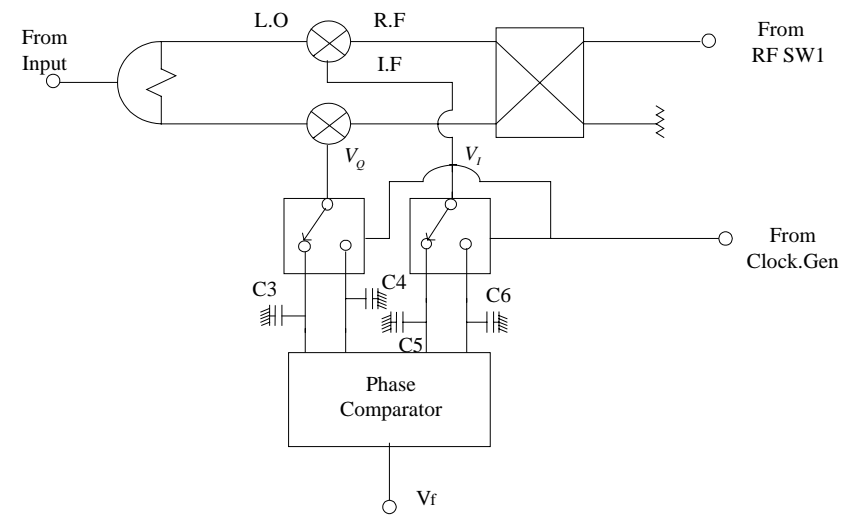

Figure 2: Schematic of IQ demodulator 


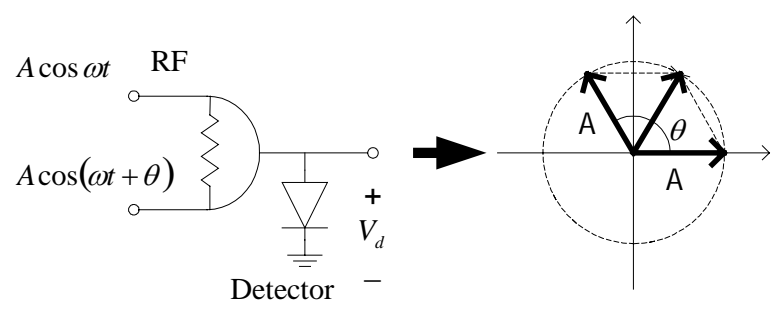

Figure 3: Controller schematic of phase controller

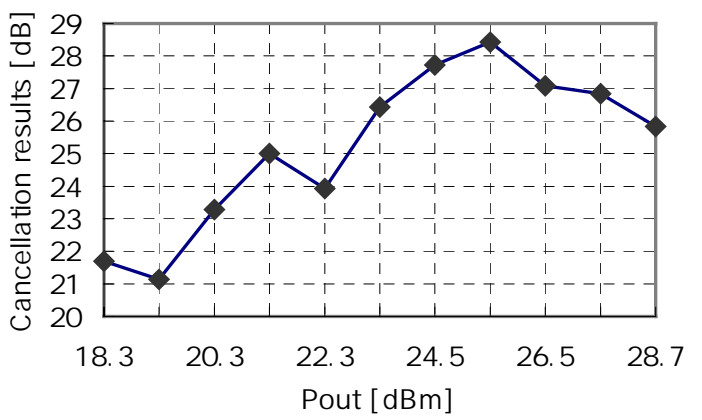

Figure 4: Adaptive main signal cancellation results

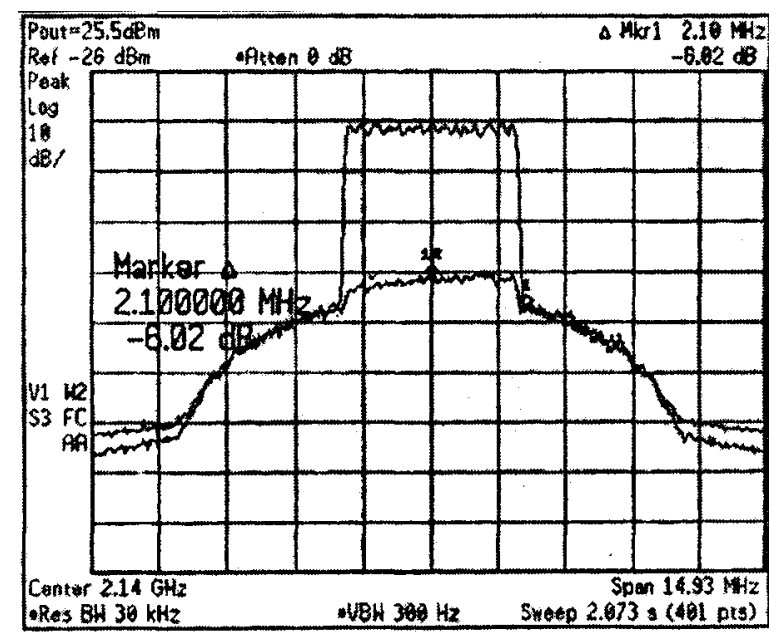

Figure 5: Main signal cancellation result (@Po=25.5dBm)

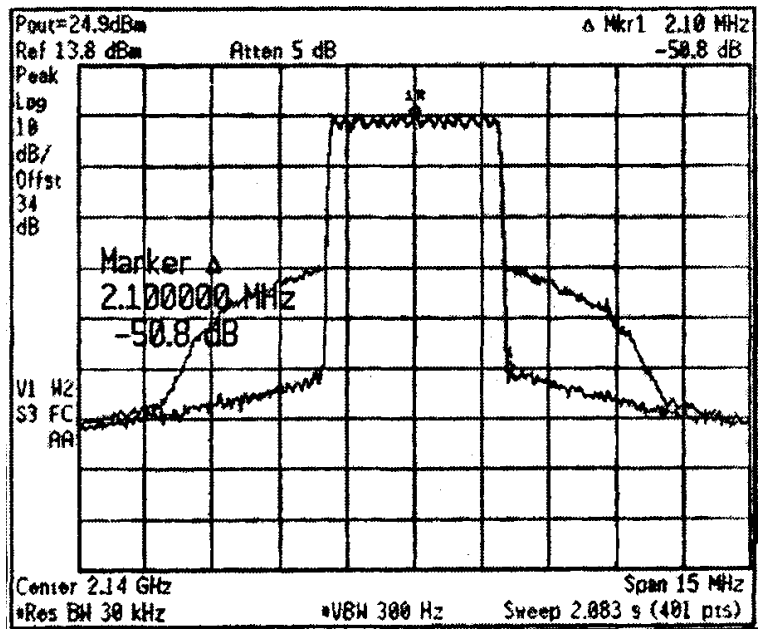

Figure 6: Linearization result (@Po=24.9dBm)

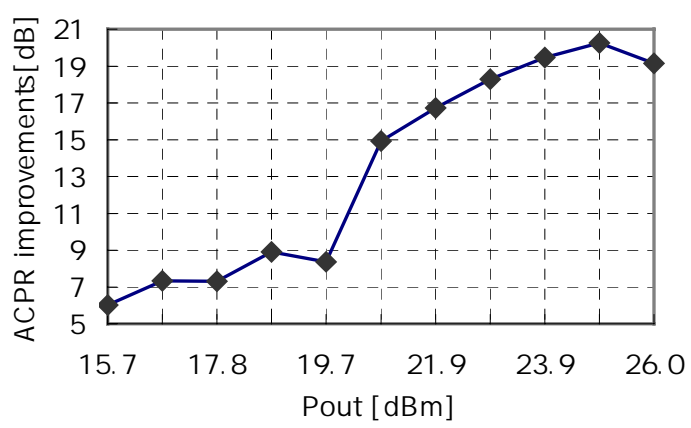

Figure 7: ACPR results with adaptive linearization 